\title{
CCD Standard Fields
}

\author{
D.H.P.Jones
}

Royal Greenwich Observatory, Cambridge

\begin{abstract}
There are several standard fields for CCDs available in the literature. Some of these have been observed many times with the CCD cameras of the Isaac Newton Group of Telescopes on La Palma. These observations were made in order to establish the zero-points and colour equations for the $U B V R I$ filters. Now that many observations are available in the data archive it is possible to combine them and search for inconsistencies in the published magnitudes and colours. This discussion will lead to standard fields of a higher quality. Furthermore astrometric plates have been taken of several of these fields which will be used to provide accurate positions as well as magnitudes and colours. These will provide the scale, orientation and distortion of any CCD camera.
\end{abstract}

\section{The Observatorio del Roque de los Muchachos}

Our observatory on the Canary Island of La Palma comprises three telescopes operated by the Royal Greenwich Observatory with apertures of 1.0, 2.5 and 4.2 metres. They are operated on the 'common user' principle for an international community including British, Dutch, Spanish and Irish astronomers; numbering about a thousand active members.

\section{The La Palma Data Archive}

Since first light in 1984 the great majority of observations have been kept in the La Palma Data Archive (Zuiderwijk 1991, Zuiderwijk and Meikle 1992). Only the unreduced data, together with attendant flat fields, bias frames and the like are held in the archive. Descriptions of the 165000 observations made to date are held in a disk file at Cambridge which can be examined from virtually any computer worldwide connected to a network.

\section{Standard Fields}

About half the observing on the 1-metre and 2.5-metre telescopes has been devoted to direct imaging and the photometric calibration fields of the six clusters observed by Christian et al (1985) and of M67 observed by Schild (1983) have been frequently observed. The archive reflects the popularity of these fields and contains the following numbers of observations: 


$\begin{array}{lr}\text { NGC 2264 } & 74 \\ \text { NGC 2419 } & 76 \\ \text { NGC } 4147 & 138 \\ \text { M92 } & 422 \\ \text { NGC 7006 } & 298 \\ \text { NGC 7790 } & 221 \\ \text { M67 } & 730\end{array}$

\section{The M92 Field}

As part of our support work for the La Palma telescopes I have embarked on an examination of these data. I discuss here only the $R, I$ observations of M92 which are typical of the whole. A comparison between $R$ magnitudes based on 10 exposures with a GEC chip on the Jacobus Kapteyn 1-metre telescope over a week in 1992 June and those published by Christian et al. is shown in Fig.1.

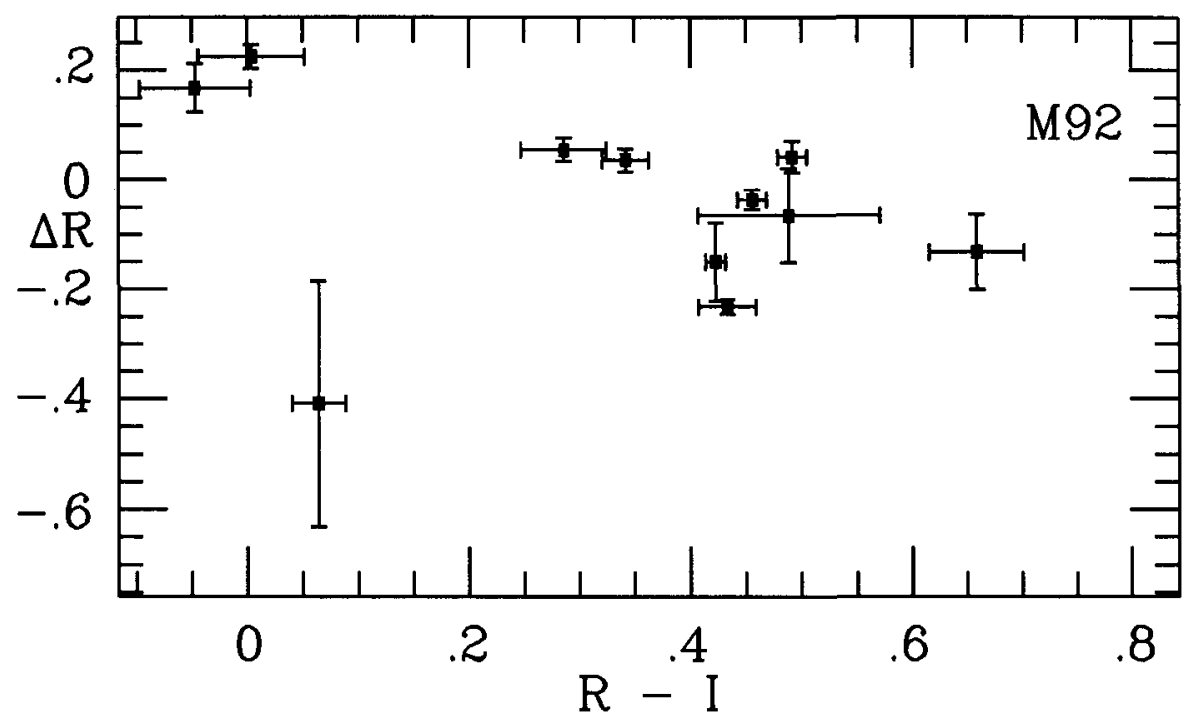

Figure 1 Difference in $R$ (Jones - Christian) for stars in M92 plotted as a function of $(R-I)$ Christian.

The CCD observations were reduced with simple aperture photometry in a $10 \operatorname{arcsec}$ diaphragm to make them directly comparable with the photomultiplier observations of Christian et al. The median error of the CCD observations which total $640 \mathrm{sec}$ is 0.011 mag compared to 0.029 for Christian et al. Even making due allowance for the errors, the two series do not agree well; in fact they imply a colour equation $\sim 0.4(R-I)$. The origin of this colour equation remains a mystery because the 
colour equation for this chip-filter combination is given as $0.045(R-I)$ by Unger et al. (1988); based on a comparison with the work of Schild (1983) in M67 with a scatter of $\pm 0.009 \mathrm{mag}$.

\section{The Kron-Cousins System}

The La Palma $R, I$ photometry is designed to be on the Kron-Cousins system. Christian et al. nowhere explicitly state what their system is. However they used the filters recommended by Bessell (1979) and the standard stars of Landolt (1983), both of which refer to the Kron-Cousins system. Fig.2 shows a comparison between the La Palma CCD $(R-I)$ colours and those of Christian et al.

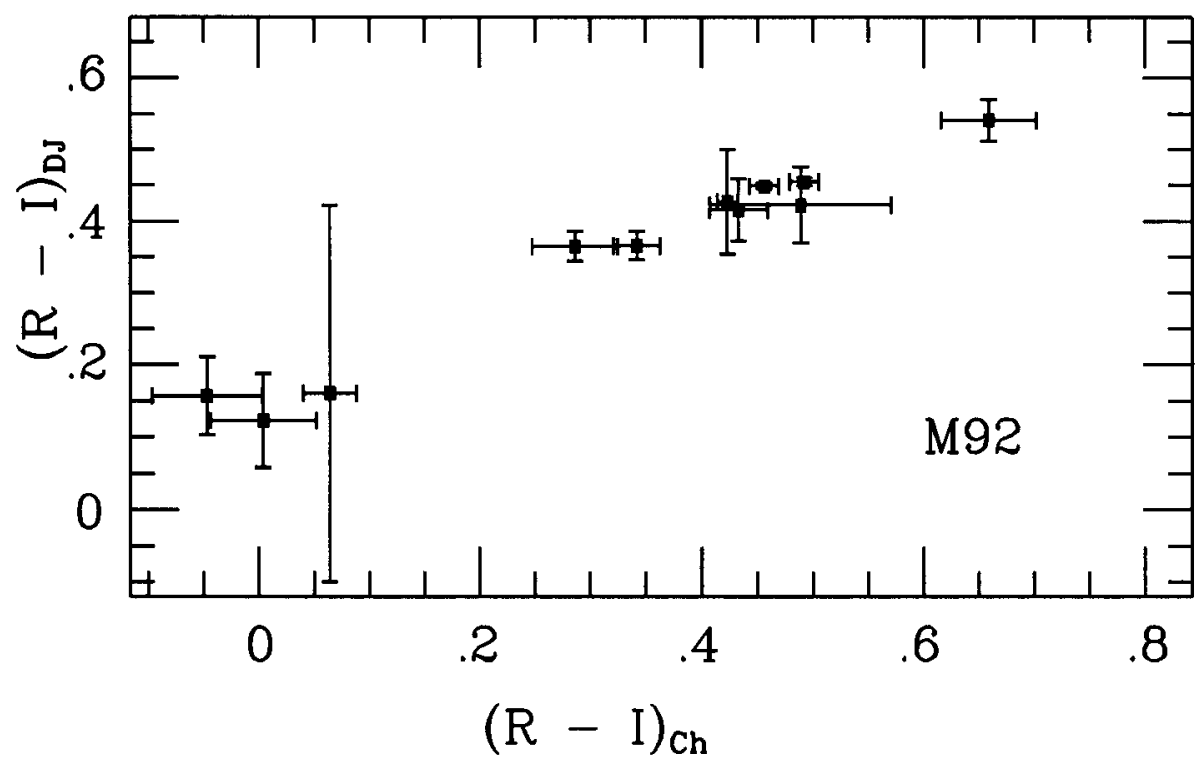

Figure $2(R-I)$ (Jones) plotted against $(R-I)$ (Christian) for stars in M92.

The observations agree within their errors but there is a clear systematic difference.

\section{Flatfielding}

There is always the possibilty that the scatter in Fig. 1 and 2 is caused by some defect in the process of flatfielding. Our usual procedure on La Palma is to use the twilight sky on a field chosen to be devoid of bright stars. Faint stars are eliminated by offsetting the telescope by a few arcseconds between exposures and taking the median of series of exposures, sometimes continuing over several evenings and mornings. The marginal distributions in sensitivity of the $R$ flatfield used in reducing the observations 
in Fig.1 is shown in Fig.3.

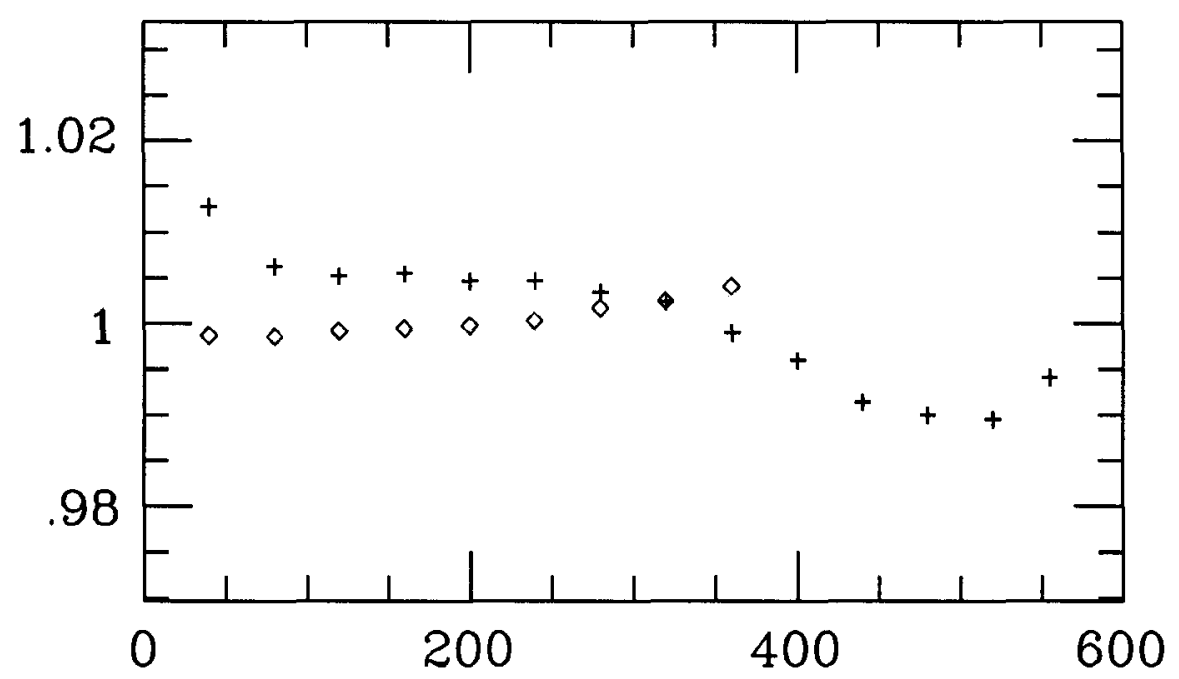

Figure 3 Marginal averages of a typical median twilight flatfield in $R$. The diamonds are along the rows $40-360$ and the crosses along the columns 40-560; each symbol embraces 40 rows or columns.

It is difficult to believe that failure to flatfield properly can have introduced more than a scatter of a few thousandths of a magnitude into Fig.1.

\section{Astrometric Calibration}

The reason for studying these fields in detail is to improve the internal accuracy and to extend the photometry to fainter limits suitable for larger telescopes. Some visiting observers require astrometric calibration as well as photometric of their CCD observations. There are little suitable data in the modern literature so I have recently taken photographic plates with our 1-metre telescope of all these fields. However I have also found that the bifilar micrometer measures by Barnard (1931) and the photographic observations of Schlesinger (1934) give comparable results.

\section{References:}

Barnard, E.E., 1931, Pub.Yerkes Obs., 6.

Bessell, M.S., 1979, Pub. Astr. Soc. Pacific, 91, 589.

Christian, C.A., Adams, M., Barnes, J.V., Butcher, H., Hayes, D.S., Mould, J.R. and Siegel,M., 1985, Pub. Astr. Soc. Pacific, 97, 363.

Landolt, A.U., 1983, Astron. J., 88, 439.

Schild, R.E., 1983, Pub. Astr. Soc. Pacific, 95, 1021. 
Schlesinger, F., 1934, Astron. J., 44, 21.

Unger, S.W., Brinks, E., Laing, R.A., Tritton, K.P. and Gray,P.M., 1988 La Palma Observers' Guide, $\mathrm{p} 86$.

Zuiderwijk, E.J., 1991, La Palma Technical Note No.69.

Zuiderwijk, E.J. and Meikle, W.P.S., 1992, Gemini, 36, 12.

\section{Discussion}

W. Tobin: Do you see any evidence for distortion in your CCD cameras?

Jones: Not down to the one tenth of a pixel level but there may be smaller distortions which we haven't been able to detect.

T.J. Kreidl: With very large telescopes $(8-10 \mathrm{~m})$ coming on-line this decade, the need for faint standards is very important. Please comment on your efforts to improve the present evident discrepancy between the standard calibrations you are comparing your data with, and what precision you hope to achieve for, say, 16-18 magnitude standards.

Jones: The immediate aim is to improve the internal consistency of the Christian et al sequences which are around magnitude 16. It is difficult to use archive data to check the external accuracy of the work of Christian et al. I agree that it is very important to provide standards for the new generation of large telescopes.

E. Budding: My impression was that the chief contribution to the large discrepancies you show is related primarily to the filters. This was presumably the KPNO set which were commented on previously. This point gave rise to thoughts about the role of another cut-off effect which may have an important bearing on filter choice, ie the financial cut-off. Is this speculation relevant to your situation?

Jones: We are in the process of replacing the KPNO interference filters with the Harris recipe for combinations of Schott glasses, published in the Kitt Peak newsletter about 3 years ago. these glasses are comparatively cheap and so we aren't constrained financially.

R. Florentine-Nielsen: Was the $\Delta R$ a function of the $R$ magnitude itself, i.e. did you test for any nonlinearity in your $C C D$ photometry?

Jones: At the beginning of each CCD run we routinely check the linearity of the CCD with a constant light source and exposures of increasing length. This does not verify the linearity of the CCD in the exact sense of your question but we find the number of electrons is linearly related to exposure time up to $50 \mathrm{~K}$ for all our chips, and $64 \mathrm{~K}$ for some of them. As a by-product we also derive the ratio of electrons to $\mathrm{ADU}$ and the read-out noise. 


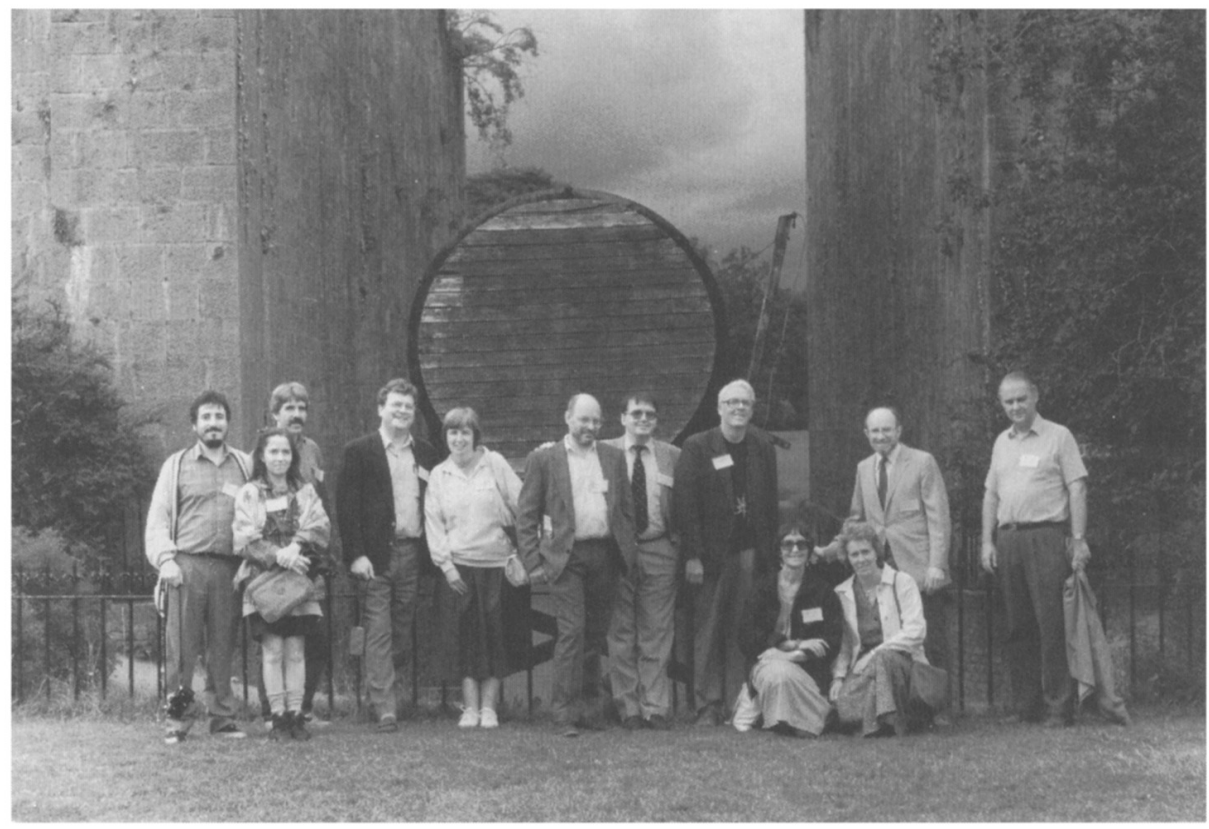

A group standing in front of the remains of The Leviathan of Parsonstown, the great six foot reflecting telescope built by the fourth Earl of Rosse in the grounds of Birr Castle, Co. Offaly, in 1845. 